\title{
Aziridinas e a sua conversão assimétrica em compostos bioativos
}

\author{
Saúl Silva, ${ }^{1}$ Christopher Maycock ${ }^{1,2}$ \\ ${ }^{1}$ Instituto de Tecnologia Química e Biológica António Xavier, Universidade Nova de Lisboa \\ ${ }^{2}$ Departamento de Química e Bioquímica, Faculdade de Ciências, Universidade de Lisboa \\ saulsilva@itqb.unl.pt; maycock@itqb.unl.pt
}

\begin{abstract}
Aziridines and their asymmetric conversion to bioactive compounds - This article describes the application of enzymatic resolution to the formation of optically active azabicyclo[x.1.0]alkanes (bicyclic aziridines) and their use for the synthesis of biologically active molecules. The commercial antiviral agent oseltamivir and its phosphorus containing analogue tamiphosphor were prepared from this starting material $(x=4)$. This route to antiviral analogues is applicable to the synthesis of a range of oseltamivir analogues. A similar aziridine $(x=3)$ was used as starting material for studies related to the stereoselective synthesis of the natural product terpestacin. Although the overall synthetic strategy was not successful some interesting reactions and compounds were encountered. Also new methods and reagents for the preparation of alfa-chlorocarbonyl compounds and 1,3-diketones or 1,3-ketoacid derivatives were developed during these studies.
\end{abstract}

Este artigo descreve a preparação de azabiciclo[x.1.0]alcanos (aziridinas bicíclicas) através de resolução enzimática e o seu uso na síntese de moléculas bioativas. O oseltamivir, um antiviral comercial, e o seu análogo contendo fósforo, o tamifósforo (tamiphosphor), foram preparados usando estes compostos $(\mathrm{x}=4)$ como material de partida. Esta nova via de síntese poderá ser usada na preparação de novos análogos do oseltamivir com potencial atividade contra vírus influenza. Uma aziridina similar $(\mathrm{x}=3)$ foi usada como material de partida em estudos relacionados com a síntese estereosseletiva da terpestacina, um produto natural. Apesar da estratégia não ter tido sucesso, foram identificadas algumas reações e moléculas interessantes. Também foram estabelecidos neste trabalho alguns métodos e reagentes para a preparação de compostos $\alpha$-clorocarbonílicos e 1,3-dicetonas ou derivados de 1,3-cetoácidos.

Os compostos orgânicos são necessários no dia a dia (medicamentos, aditivos alimentares, tintas, polímeros, etc.) e em diferentes áreas científicas (biologia, materiais, farmácia, etc.). Consequentemente, a síntese orgânica tem um papel importante na melhoria da nossa qualidade de vida e no desenvolvimento da Ciência. Por um lado, tornando disponíveis novas moléculas com potencial utilidade para a sociedade e, por outro, criando novos métodos de síntese mais eficientes e verdes.

"Why do chemists make molecules? The obvious (and true) answer is: because we need them. That is why chemical synthesis is still vibrant, and will continue to supply the drugs, materials and commodities of the twenty-first century. Every year brings its bounty.” [1].

Em geral, estratégias sintéticas são constituídas por vários passos para transformar o material de partida no produto final, cada um destes passos consistindo numa reação ou transformação química. O desenvolvimento de reações novas e mais eficientes é essencial para o progresso da química orgânica, dado que estas são as ferramentas que um químico possui para moldar moléculas com a estrutura pretendida. Quanto mais ferramentas estiverem disponíveis, maior será o número de moléculas possíveis de preparar. Apesar de alguns grupos focarem a sua investigação exclusivamente ou no desenvolvimento de novas reações ou na síntese de novas moléculas, as duas estão intrinsecamente ligadas. No desenvolvimento de uma nova reação, a sua utilização numa síntese complexa demonstra a sua utilidade ou, porventura, as suas limitações. Por outro lado, uma nova rota de síntese pode implicar o desenvolvimento de um novo método para efetuar uma determinada transformação.

Estratégia para preparação de aziridinas quirais oticamente ativas

Muitos fármacos quirais são comercializados como um único enantiómero, por isso a síntese enantiosseletiva é um tópico importante da síntese orgânica. Uma estratégia frequente é o uso de um material de partida quiral enantiomericamente puro. Alguns compostos deste tipo são comercializados a um custo acessível, sendo normalmente de origem natural ou produzidos por fermentação, como por exemplo ácidos simples (e.g. ácido tartárico), aminoácidos ou açúcares. Como alternativa, materiais de partida mais disponíveis aquirais ou racémicos podem ser usados quando submetidos a uma reação enantiosseletiva, tendo sido esta a estratégia utilizada nas sínteses descritas neste artigo.

Uma outra possível estratégia para a preparação de moléculas oticamente ativas é a resolução de um racemato, que consiste na separação dos dois enantiómeros presentes numa mistura. Diferentes metodologias podem ser usadas para este fim: cristalização seletiva, derivatização quiral, cromatografia quiral ou resoluções cinéticas. Algumas resoluções cinéticas podem ser um processo enzimático, estando descritas múltiplas resoluções enzimáticas em aplicações laboratoriais e industriais [2]. A resolução de álcoois secundários com uma lipase é provavelmente um dos métodos mais usados, cuja reação consiste numa acilação seletiva de um dos enantiómeros. Este processo é relativamente económico e geralmente eficiente, sendo 

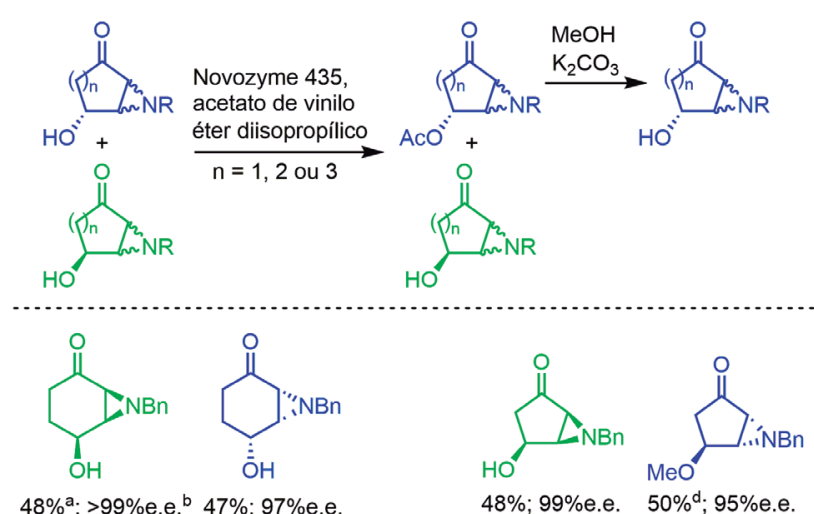

$48 \%{ }^{\text {a }}$; $99 \%$ e.e. ${ }^{\text {b }} 47 \%$; $97 \%$ e.e. $E^{\mathrm{c}}>200$

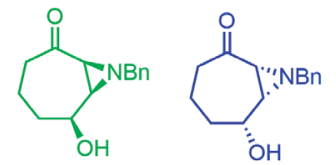

$48 \%$; $92 \%$ e.e. $\quad 50 \%$; $93 \%$ e.e. $E=82$

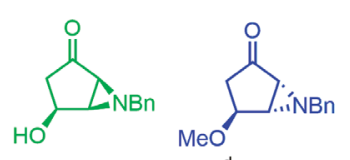

$48 \%$; $99 \%$ e.e. $\quad 50 \%$ d ; $95 \%$ e.e. $E>200$<smiles>COC1CCC(=O)C2CCCCC12</smiles>

$46 \%$; $91 \%$ e.e. $\quad 49 \%$; $96 \%$ e.e. $E=83$

a Os rendimentos apresentados foram calculados com base na massa dos produtos purificados. ${ }^{\mathrm{b}}$ e.e. corresponde aos excessos enantioméricos dos produtos e estes valores foram calculados através de HPLC (quiral). ${ }^{\mathrm{c}} \mathrm{E}$ representa o valor de proporção enantiomérica e foi calculado com base no valor de e.e. dos dois produtos. ${ }^{\mathrm{d}} \mathrm{Na}$ metanólise ocorreu a eliminação de acetato e a adição de metanol.

Esquema 1 - Resolução enzimática de 4-hidroxiacilaziridinas cíclicas.

a recuperação e reutilização da enzima, quando possível, uma grande vantagem. A lipase B de Candida antarctica é comercializada em suporte sólido com o nome Novozym 435 e é amplamente usada devido ao seu fácil manuseamento, grande espetro de substratos e fácil recuperação, tendo sido também usada no trabalho descrito neste artigo.

Recentemente desenvolvemos um novo método de preparação de aziridinas oticamente ativas que inclui uma resolução enzimática muito eficiente (Esquema 1) [3]. É um método acessível, reprodutível e escalável que tem potencial para ser usado industrialmente. Constitui uma estratégia útil para a produção de materiais de partida oticamente ativos para a síntese estereosseletiva de cicloalcanos que contêm nitrogénio. O esquema 2 ilustra possíveis transformações químicas destas aziridinas e, deste modo, a sua versatilidade em síntese orgânica.

\section{Tentativas de síntese da terpestacina}

A terpestacina é um sesterterpeno produzido por diversos fungos, que foi descrito como tendo atividade antiviral [4] e anticancerígena [5]. Tendo em conta o potencial desta molécula como drug lead e dada a complexidade da sua estrutura, tem sido um alvo para químicos sintéticos e uma das sínteses mais expeditas foi desenvolvida por Trost e colaboradores [6] (Esquema 3). Apesar de a formação do macrociclo presente na molécula apresentar alguns desafios, a construção do ciclopentano é provavelmente a tarefa mais difícil, já que requer um grande controlo da estereoquímica dos grupos presentes. Outro dos objetivos deste trabalho era desenvolver uma nova síntese do intermediário de Trost e possivelmente da terpestacina, usando como material de partida uma das aziridinas obtidas pelo método anterior.

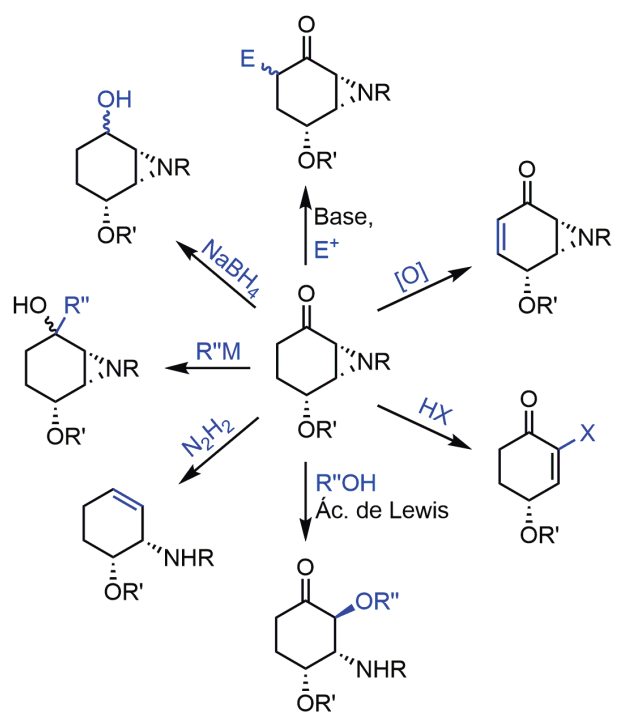

Esquema 2 - Exemplos de reações de 4-hidroxiacilaziridinas cíclicas.

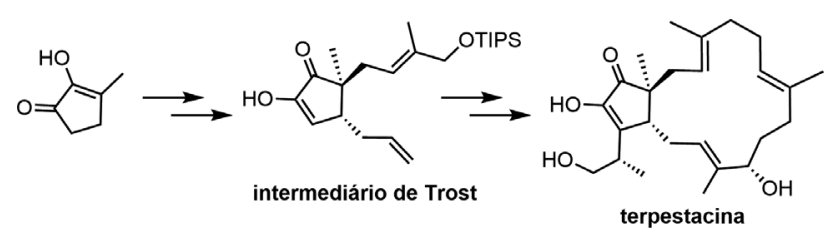

Esquema 3 - Representação genérica da síntese da terpestacina pelo processo de Trost [6].

O esquema 4 ilustra a estratégia desenhada para a síntese do intermediário de Trost. Nesta estratégia, a estereoquímica dos grupos é orientada pela aziridina, em que o primeiro grupo alilo seria adicionado em posição trans relativamente à azidiridina e o segundo grupo alilo mais substituído trans ao primeiro. Numa primeira tentativa, a adição de Michael ocorreu de forma seletiva (Esquema 5), tal como previsto, contudo a metilação do enolato não foi possível. Numa segunda tentativa, usou-se um material de partida que já possuía o metilo na posição pretendida. Contudo, apesar da adição de Michael ter ocorrido, os dois anéis do sistema bicíclico colapsaram, dando origem a um produto linear. Realizou-se ainda uma terceira tentativa com um material de partida com um metilo numa posição diferente, sendo que o segundo grupo alilo seria introduzido não por alquilação, mas por abertura da aziridina e rearranjo de Claisen. Contudo esta última estratégia também não teve sucesso, já que a adição de Michael originava um rendimento baixo (30\%) e nenhuma estereosseletividade. Como conclusão, o insucesso da estratégia delineada parece ser devido à elevada tensão criada pelo sistema bicíclico e ao facto de este não suportar os três grupos alquilo.

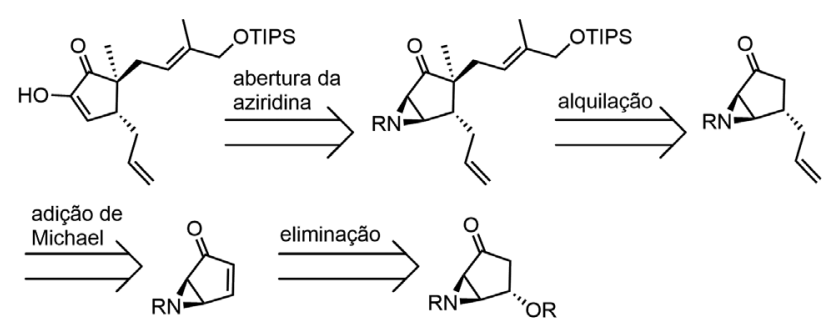

Esquema 4 - Retrossíntese do intermediário de Trost. 

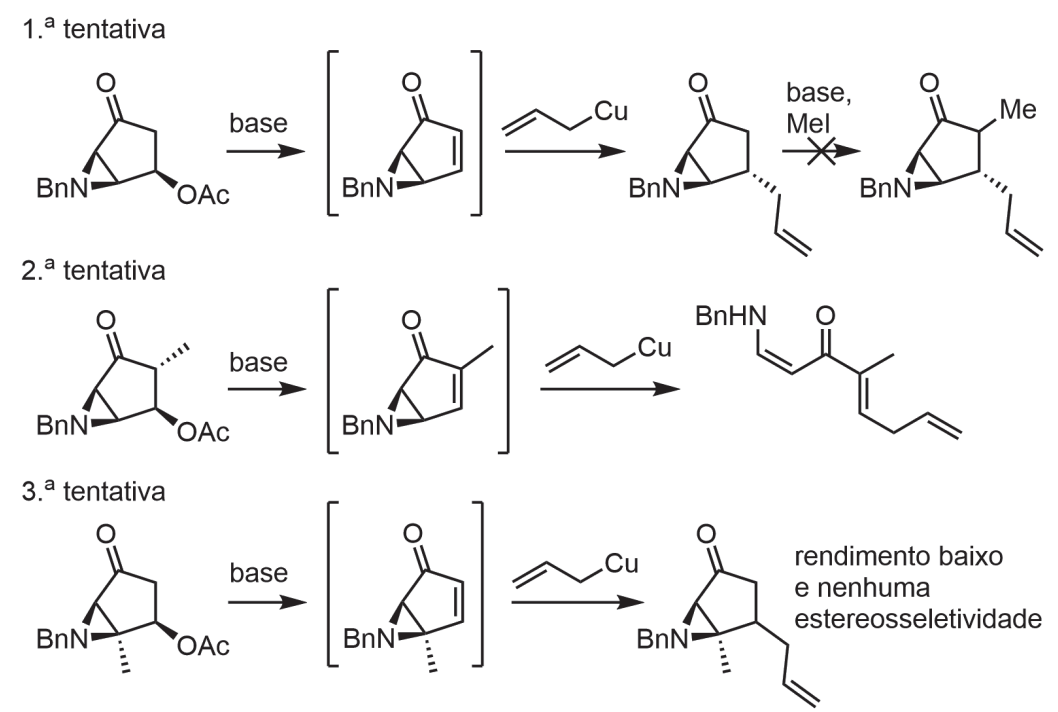

Esquema 5 - Tentativas de síntese do intermediário de Trost.

Numa das aziridinas preparadas nas tentativas de síntese descritas anteriormente foi possível observar invertómeros por ${ }^{1} \mathrm{H}$ RMN (Figura 1) e ${ }^{13} \mathrm{C}$ RMN. Invertómeros são um tipo especial de confórmeros que, no caso de compostos com nitrogénio, correspondem à sua inversão (efeito guarda-chuva). Na maior parte dos compostos nitrogenados esta inversão é tão rápida que não é possível observar a presença de invertómeros usando técnicas espetroscópicas. No entanto, existem algumas exceções, por exemplo, quando um dos substituintes do nitrogénio é um átomo eletronegativo (e.g. cloro ou oxigénio), este equilíbrio é mais lento e em alguns casos é mesmo possível isolar os invertómeros. Dentro do nosso conhecimento, nunca foram reportados invertómeros de alquilaziridinas e, dentro da experiência do nosso grupo, é também a primeira vez que observamos este tipo de confórmeros em aziridinas. Para perceber melhor este fenómeno, realizámos cálculos DFT (teoria do funcional da densidade) e concluímos que, na grande maioria das aziridinas, o confórmero endo tem uma energia muito mais alta que o exo, não sendo por isso detetável por RMN. Na aziridina da figura 1, a dupla ligação e o grupo metilo parecem destabilizar o confórmero exo, aproximando a sua energia do endo, e sendo assim possível observar os dois invertómeros.

\section{Síntese de oseltamivir e análogos}

As infeções causadas pelo vírus da gripe continuam a ser um risco para a saúde humana na sociedade moderna. Derivados de ácidos siálicos e moléculas estruturalmente equivalentes têm sido preparados e demonstraram ter uma considerável atividade antiviral através de inibição de enzimas essenciais ao desenvolvimento de infeções virais. O fármaco mais usado no tratamento destas infeções é o oseltamivir que foi desenvolvido pela Roche e comercializado com o nome Tamiflu ${ }^{\circledR}$.

O processo inicialmente usado pela Roche usava o ácido xiquímico como material de partida, um produto natural quiral oticamente puro. A principal fonte de ácido xiquímico é o anis estrelado, uma planta cultivada maioritariamente na Ásia e por isso, toda a produção de Tamiflu estava dependente da disponibilidade desta planta. Consequentemente, em 2006 [7] foi especulado que o processo não teria capacidade de produzir Tamiflu suficiente para as necessidades globais em caso de pandemia, o que se veio a verificar em 2009. O desenvolvimento de novos processos tornou-se um alvo de investigação para químicos e novas sínteses continuam a ser desenvolvidas ainda hoje.

Como o vírus da gripe continua a sofrer mutações, estirpes resistentes ao oseltamivir começam a aparecer, ainda que esporadicamente. É por isso importante o desenvolvimento de antivirais alternativos. Em 2012 foi descoberta uma nova substância ativa: o tamifósforo [8]. Apesar desse composto ter demonstrado ser muito potente in vitro contra estirpes resistentes, a sua baixa biodisponibilidade é um entrave à sua utilização clínica no tratamento da gripe.

O oseltamivir e o tamifósforo são inibidores da enzima neuraminidase (NA) do vírus da gripe. A NA viral cliva o ácido siálico presente na superfície das células infetadas,

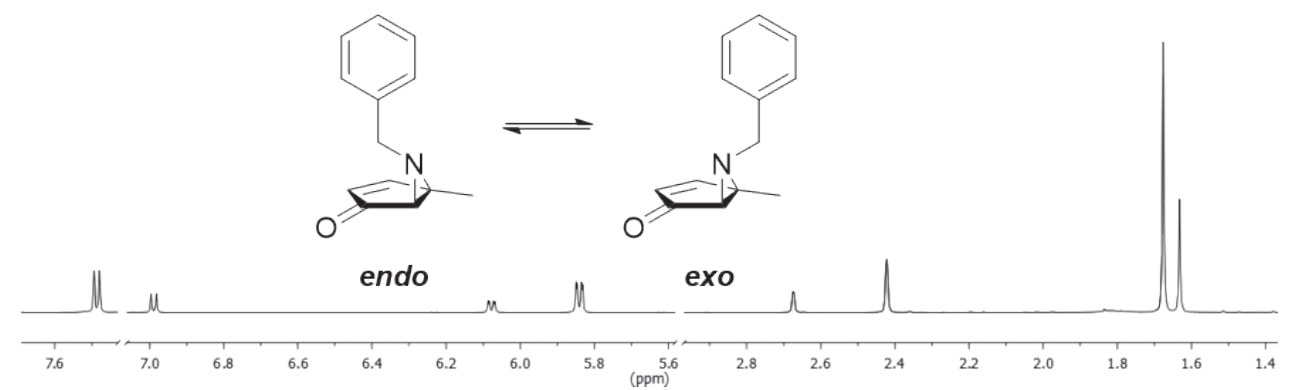

Figura 1 - Espetro de ${ }^{1} \mathrm{H}$ RMN parcial da 6-benzil-5-metil-6-azabiciclo[3.1.0]hex-3-en-2-ona, em que é possivel observar os sinais relativos aos seus invertómeros, que se encontram representados em equilíbrio. 
facilitando a propagação do vírus (Esquema 6). O oseltamivir é um pró-fármaco que é hidrolisado a ácido in vivo, sendo este último a espécie bioativa (Esquema 7). Em ambos os fármacos, a estereoquímica dos grupos funcionais presentes na molécula é essencial à sua atividade. Em todas as sínteses do oseltamivir e outros inibidores da NA, é crucial um grande controlo da estereoquímica dos carbonos assimétricos no produto final.

Outro objetivo deste trabalho era desenvolver sínteses versáteis de substâncias antivirais para tratamento da gripe. Pretendia-se, por um lado, permitir a produção de oseltamivir usando matérias-primas alternativas e, por outro, a obtenção de novos análogos potencialmente ativos.

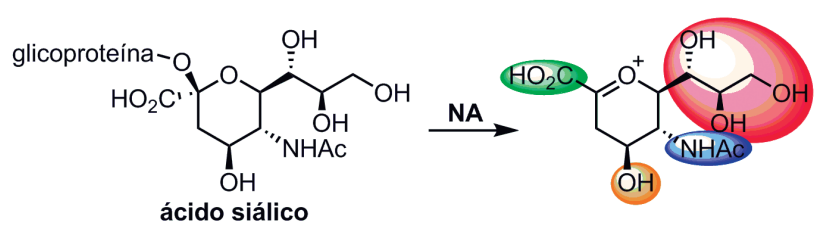

Esquema 6 - Hidrólise do ácido siálico presente em células infetadas pelo vírus da gripe, catalisada pela neuraminidase (NA) viral.
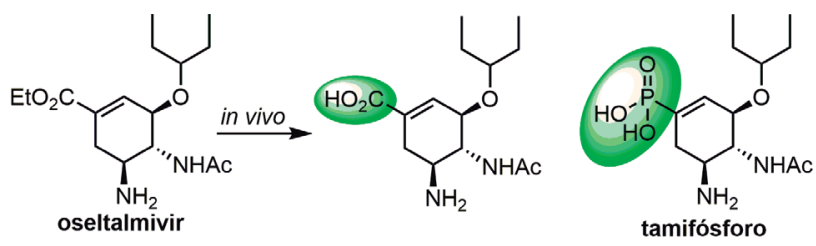

Esquema 7 - Hidrólise in vivo do pró-fármaco oseltamivir e formação do respetivo ácido, espécie bioativa, e estrutura do seu análogo tamifósforo. Os principais grupos funcionais responsáveis pela atividade biológica destes dois compostos estão evidenciados com diferentes cores.
Neste trabalho, foram desenvolvidas novas sínteses formais dos antivirais oseltamivir e tamifósforo (Esquema 8) [9]. Recorrendo ao novo método de resolução enzimática, uma aziridina quase enantiomericamente pura foi preparada e usada como material de partida. Esta aziridina pode ser preparada a partir do anisol ou ciclo-hexadieno, dois materiais de partida muito mais abundantes e económicos quando comparados com o ácido xiquímico.

A formação estereosseletiva da aziridina na 4-hidroxiciclo-hex-2-enona protegida é uma das características mais distintas de outras descritas anteriormente. $\mathrm{O}$ anel da aziridina controla a estereoquímica de todos os centros estereogénicos formados na molécula: o grupo isopentiloxilo, trans relativamente ao grupo acetamido, formado através da abertura do anel da aziridina; e o grupo amino, também trans relativamente ao grupo acetamido, que é formado por inversão do grupo álcool.

Outra característica importante da síntese é a introdução do grupo éster ou fosfonato num enolato de lítio. Os 3-oxo éster ou fosfonato produzidos são depois convertidos no éster insaturado presente no produto final. Neste sentido, novos análogos do oseltamivir ou tamifósforo podem ser preparados usando esta metodologia, dado que diferentes grupos funcionais podem ser introduzidos no lugar do ácido carboxílico. Podem assim ser preparadas novos compostos com melhores propriedades biofarmacêuticas, nomeadamente, com biodisponibilidade adequada.

\section{$\alpha$-Funcionalização de compostos carbonílicos}

Na tentativa de preparar novos análogos do oseltamivir com diferentes bioisósteros do grupo ácido carboxílico, foram identificadas e estudadas duas reações. Estas duas rea-
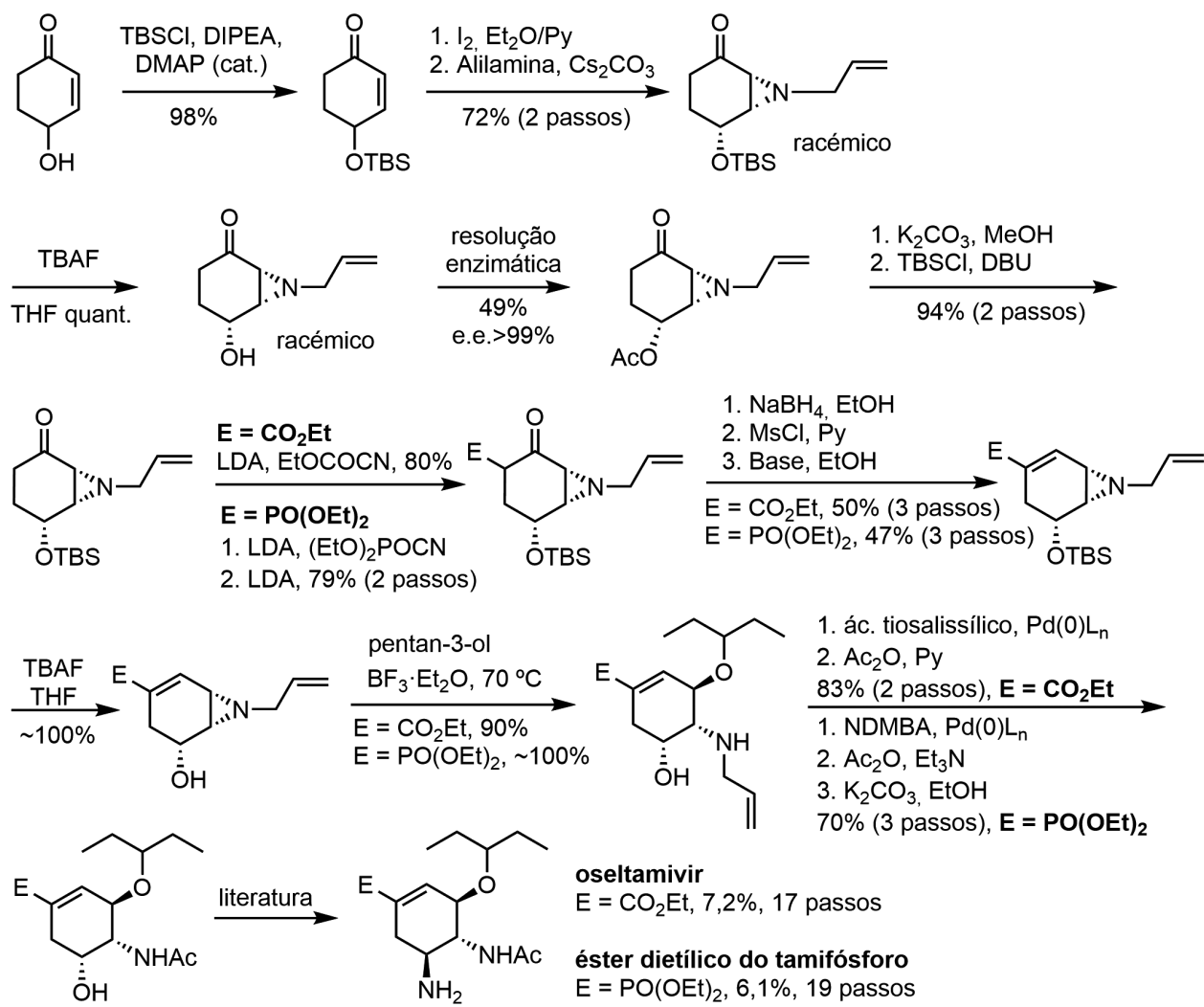

Esquema 8 - Síntese do oseltamivir e do tamifósforo a partir de 4-hidroxiciclo-hex-2-enona. A conversão do grupo hidroxilo em amino encontra-se descrita na literatura. 


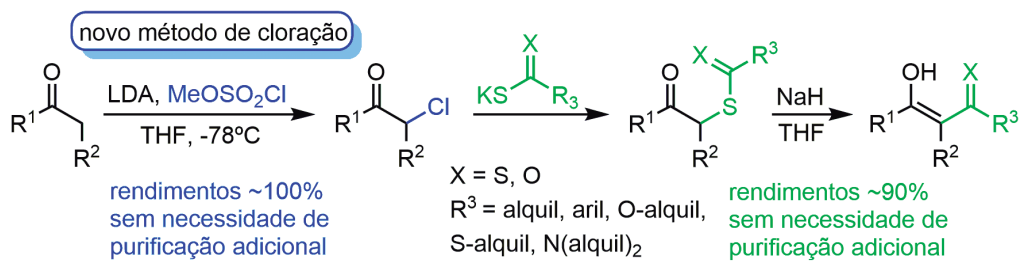

Esquema 9 - Reações de $\alpha$-funcionalização de compostos carbonílicos: cloração e extrusão de enxofre.

ções permitem a funcionalização da posição $\alpha$ a um grupo carbonilo de forma eficiente, nomeadamente em cetonas (Esquema 9).

Uma das reações é a cloração em meio básico usando clorossulfato de metilo [10], sendo este reagente descrito pela primeira vez como uma fonte eletrofílica de cloro. A outra consiste num rearranjo com extrusão de enxofre em meio básico, em que ocorre a transformação de compostos 2-oxo-S-(tio)carbonílicos em 2-oxotiocarbonílicos. As duas reações são altamente eficientes e limpas, não havendo necessidade de purificação adicional depois do work-up. A reação de cloração é particularmente útil para a transformação de compostos sensíveis a meio ácido, já que os métodos descritos para este tipo de halogenações são maioritariamente em meio ácido. Por outro lado, durante a reação de extrusão de enxofre é formada uma nova ligação carbono-carbono, uma das transformações mais importantes em síntese orgânica, constituindo assim numa nova estratégia sintética que tem sido pouco explorada.

\section{Perspetivas futuras}

Em todas as estratégias descritas, o objetivo foi criar metodologias eficientes, reprodutíveis e que usam reagentes pouco tóxicos e acessíveis, de modo a serem facilmente usadas por outros químicos sintéticos, mesmo com pouca experiência. As estratégias sintéticas descritas neste trabalho podem ser usadas na preparação de diferentes cicloalcanos nitrogenados quirais, em particular de ciclo-hexanos e ciclopentanos. Tornam assim possíveis novas vias de síntese para diferentes moléculas com potencial atividade biológica, nomeadamente antiviral. Em última análise, estas novas moléculas poderão mostrar potencial para serem inseridas em programas de descoberta, planeamento e desenvolvimento de novos fármacos antivirais.

\section{Agradecimentos}

À Fundação para a Ciência e a Tecnologia (FCT) pela bolsa de doutoramento SFRH/BD/84309/2012. A unidade de investigação, projeto LISBOA-01-0145-FEDER-007660 (Microbiologia Molecular, Estrutural e Celular), foi financiada por fundos FEDER através do COMPETE2020 e por fundos nacionais através da FCT. As experiências de RMN foram realizadas no CERMAX, ITQB-NOVA, com equipamentos financiados pela FCT, projeto AAC 01/SAICT/2016.

\section{Referências}

[1] P. Ball, Nature 528 (2015) 327-329.

[2] V. Gotor-Fernández, R. Brieva, V. Gotor, J. Mol. Catal. B: Enzym. 40 (2006) 111-120.

[3] S. Silva, C.D. Maycock, Org. Chem. Front. 4 (2017) 1620 1623.

[4] M. Oka, S. Limura, Y. Narita, T. Furumai, M. Konishi, T. Oki, Q. Gao, H. Kakisawa, J. Org. Chem. 58 (1993) 1875 1881.

[5] K.C. Park, S.H. Choi, Pediatr. Surg. Int. 29 (2013) 13271340.

[6] B.M. Trost, G. Dong, J.A. Vance, J. Am. Chem. Soc. 129 (2007) 4540-4541.

[7] V. Farina, J.D. Brown, Angew. Chem. Int. Ed. 45 (2006) 7330-7334.

[8] T.-J.R. Cheng, S. Weinheimer, E.B. Tarbet, J.-T. Jan, Y.-S.E. Cheng, J.-J. Shie, C.-L. Chen, C.-A. Chen, W.-C. Hsieh, P.W. Huang, W.-H. Lin, S.-Y. Wang, J.-M. Fang, O.Y.-P. Hu, C.-H. Wong, J. Med. Chem. 55 (2012) 8657-8670.

[9] S. Silva, C.D. Maycock, Org. Chem. Front. 4 (2017) 236240.

[10] S. Silva, C.D. Maycock, Tetrahedron Lett. 59 (2018) 1233 1238.

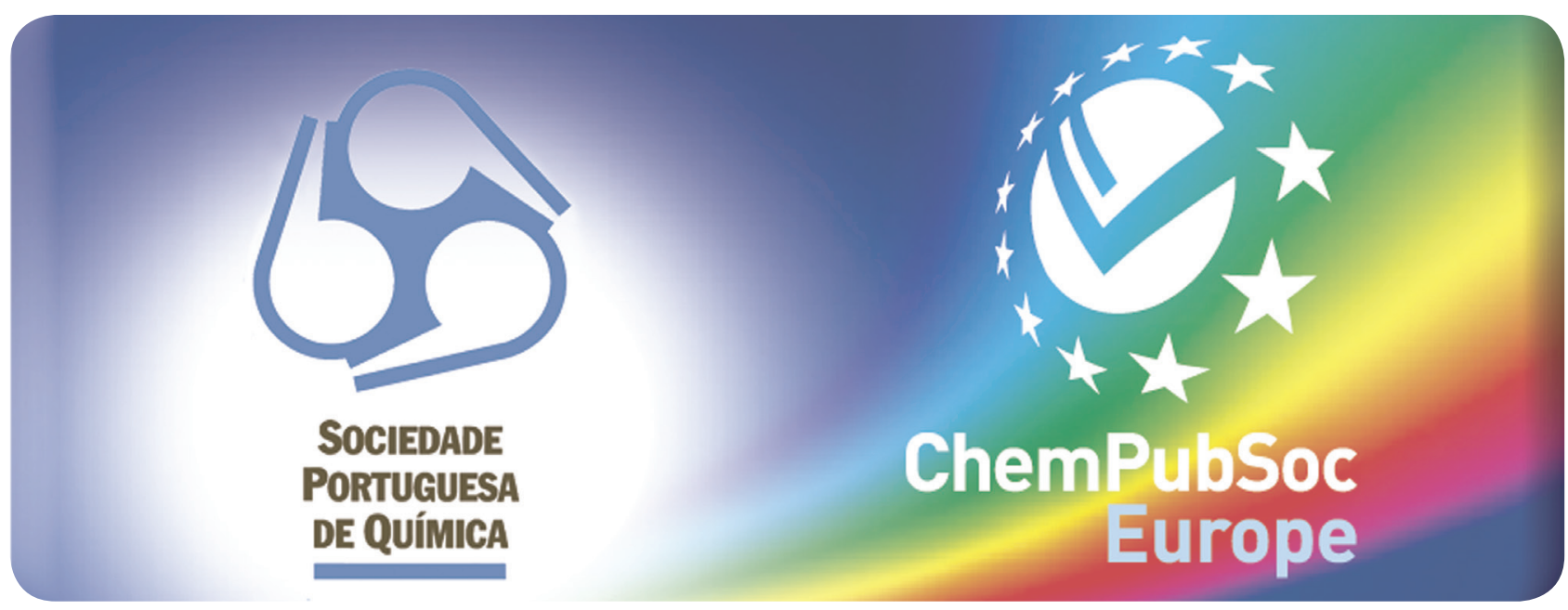

